\title{
Wie viele psychische Erkrankungen gibt es?
}

\section{Paul Hoff}

Prof. Dr. med. Dr. phil., Chefarzt, stv. Klinikdirektor, Psychiatrische Universitätsklinik Zürich, Klinik für Psychiatrie, Psychotherapie und Psychosomatik

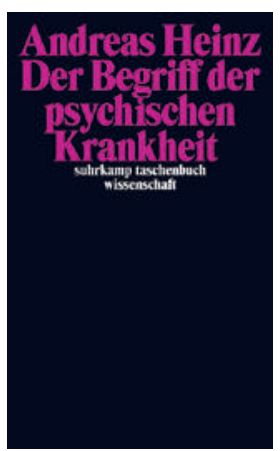

Andreas Heinz Der Begriff der psychischen Krankheit

Berlin: Suhrkamp; 2014. 371 Seiten. $25.90 \mathrm{CHF}$ ISBN 978-3-518-29708-7
Psychiatrie und Psychotherapie sind, so weit besteht ein weitgehender internationaler Konsens, nicht nur wesentliche Elemente einer jeden Gesundheitsversorgung, sie haben in jüngerer Zeit auch als attraktive und herausfordernde Forschungsfelder erkennbar an Dynamik gewonnen. Letzteres bezieht sich allerdings vorwiegend auf die empirische Forschung, vor allem auf neurowissenschaftlichem Gebiet und hier auf die komplexen Schnittstellen zwischen neuronaler Funktion und klinischem Erscheinungsbild (paradigmatisch in diesem Zusammenhang das Konzept der Endophänotypen).

Theoretische Debatten, die sich bewusst den Grundfragen unseres Faches widmen, sind hingegen weiterhin rar - ganz im Gegensatz übrigens zur psychiatrischen «Gründerzeit» zu Beginn des 20. Jahrhunderts, als in der Fachöffentlichkeit so manche theoretische Kontroverse auf hohem Niveau ausgetragen wurde. Ich meine Fragen wie die nach der Abgrenzung von psychischer Gesundheit und Krankheit, nach dem praktisch und wissenschaftlich optimalen nosologischen Modell, nach dem Stellenwert der psychiatrischen Diagnostik und, am wichtigsten, nach dem grundlegenden Verständnis von Therapie und therapeutischer Beziehung. Selbst die streckenweise irrational anmutende öffentliche Aufregung um die (angebliche) Diagnoseninflation in der 5. Version des Diagnostic and Statistical Manual of Mental Disorders (DSM-5, 2013) kann nicht darüber hinwegtäuschen, dass es um eine nachhaltige, differenzierte und breit rezipierte Auseinandersetzung mit der Theorie der Psychiatrie aktuell nicht zum Besten steht.

Genau hier setzt das Buch von Andreas Heinz an, und genau deswegen ist es ein wichtiges Buch: Es stellt die grundsätzliche Frage, was eine psychische Krankheit denn sei, oder, genauer, welchen Begriff wir uns von psychischen Krankheiten machen sollen. Es tut dies aber nicht nur in theoretischer Manier, sondern mit zahlreichen Ankerpunkten in der klinischen Praxis, um die es ja letztlich geht.

Der Autor nähert sich dem grossen Thema aus ganz unterschiedlichen Perspektiven, nämlich der klinisch- psychiatrischen, psychopathologischen, neurobiologischen, anthropologischen und philosophischen. Die souveräne Art, in der er diesen enormen Horizont erschliesst, ist bemerkenswert. Freilich wird der Text dadurch dicht und anspruchsvoll. Auch sich selbst macht es der Autor nicht leicht: Er dekliniert die Anwendbarkeit des Begriffes psychische Krankheit mit Blick auf die genannten theoretischen Perspektiven konsequent durch, ja er ringt förmlich um die plausibelste, die für den Kliniker überzeugendste Position. In den 14 Kapiteln geht es (unter anderem) um die Abgrenzung zwischen Krankheit, Kranksein und Störung (disease, illness, disorder), um das Verständnis von Krankheit als Funktionsstörung in der Innen-, Aussenund Mitwelt, um die Rolle des subjektiven Leides und die Störung sozialer Teilhabe in der Konzeption von psychischer Krankheit sowie, für Psychopathologen von besonderem Interesse, um die Relevanz von paranoider Symptomatik und von Ichstörungen für die psychiatrische Diagnostik. Detailliert erläutert der Autor die psychiatrierelevanten Positionen von vier Philosophen aus unterschiedlichen Zeitaltern und von ebenso unterschiedlicher Ausrichtung: Es geht um

\section{Das Buch stellt die grundsätzliche Frage,} was eine psychische Krankheit denn sei.

die anthropologischen Ansätze von Immanuel Kant, Max Scheler und Helmuth Plessner sowie um die Rolle von Affektion und Stimmung in Heideggers Existenzialontologie.

Um es noch einmal zu betonen: Bei aller Fülle von theoretischen Argumenten und Ansätzen findet der Autor immer wieder den Weg zurück zur klinischen Praxis und - was ihm offenbar sehr am Herzen liegt zur Kulturabhängigkeit nahezu aller menschlichen Erlebens- und Verhaltensweisen - auch solcher, die wir Psychiater als psychopathologisch auffällig oder eben als «krank» bezeichnen. Dem Rezensenten erscheint dabei das Kapitel 10, Ichstörungen und der entfremdete Selbstbezug, besonders überzeugend.

Die Zielrichtung des Buches ist eine aufklärerische: Mit selbstkritischem, aber auch selbstbewusstem 
Tenor fordert der Autor dazu auf, menschliches Verhalten nicht ohne sehr guten Grund zu pathologisieren und insbesondere sicherzustellen, dass mit psychiatrischen Diagnosen kein politischer oder sonstiger Missbrauch getrieben wird. Das aus seiner Sicht zentrale Mittel zu diesem Zweck besteht in einem eng zu fassenden, gleichsam sparsamen, aber nicht reduktionistischen Krankheitsbegriff in der Psychiatrie: Dabei

\section{Bei aller Fülle theoretischer Argumente und Ansätze findet der Autor immer wieder den Weg zurück zur klinischen Praxis.}

spürt man, vor allem in den Kapiteln über Psychosen und Suchterkrankungen, sehr wohl den grossen neurowissenschaftlichen Erfahrungshintergrund des Autors. Versuche, eine eliminative Neurowissenschaft zu etablieren, die andere Perspektiven a priori für unwissenschaftlich erklärt, weist er aber klar zurück. Es geht ihm, anders formuliert, darum, das Somatosepostulat Kurt Schneiders, das ja auch einem betont engen psychiatrischen Krankheitsbegriff verpflichtet war, um die anthropologische Dimension (einschliesslich der Subjektivität) zu ergänzen - aber eben nicht beliebig zu erweitern. Konkret läuft seine Argumentation auf eine drastische Reduktion der Zahl psychischer Erkrankungen hinaus, ein im besten Sinne radikaler Ansatz:

«Der hier vertretene Vorschlag besagt, dass eine wesentliche Störung einer psychischen Funktion immer dann als Symptom einer Krankheit zu werten ist, wenn sie das Überleben der betroffenen Person gefährdet und entweder erhebliches psychisches Leid auslöst oder die soziale Teilhabe massiv beeinträchtigt, und zwar unabhängig von ihrer Verursachung, zumindest solange die Symptome nicht bewusst inszeniert wurden und nicht jederzeit von der betroffenen Person gezielt wieder beendet werden können" (S. 137).

Am Schluss des Buches dann derselbe Sachverhalt, kliniknäher formuliert:

«Der hier formulierte Vorschlag reduziert die Zahl psychischer Erkrankungen drastisch. Statt mehrerer hundert Störungen (disorders), die derzeit in den international gültigen Klassifikationssystemen alle zumindest implizit als Krankheiten gelten, werden im hier vorgestellten Ansatz nur exogene und endogene Psychosen sowie Suchterkrankungen als psychische Krankheiten im engeren Sinne benannt ...» (S. 349).
Der kritische Impetus des Buches, seine unkonventionelle, aber profunde Herangehensweise, und dies von einem Autor, der mitten in der psychiatrischen Versorgung und Forschung arbeitet, sind ausserordentlich anregend. Auf die so angestossene Diskussion wird sicher gerade der Autor selbst gespannt sein, wird sie doch zeigen, wie weit und wohin sein Ansatz trägt. Eine mögliche skeptische Frage könnte lauten, ob durch das radikale Zurücknehmen des psychiatrischen Krankheitsbegriffes auf psychotische und Suchterkrankungen nicht ungewollt doch reduktionistischen Tendenzen Vorschub geleistet wird. Auch könnte man fragen, inwiefern bei schweren nichtpsychotischen Erkrankungen, etwa bei einer ausgeprägten Zwangserkrankung, die These, hier liege keine psychische Krankheit im engeren Sinne vor, den betroffenen Personen und ihrem Umfeld zu vermitteln wäre. Und schliesslich lässt der Text - möglicherweise sehr bewusst - die aktuell intensiv diskutierte Frage offen, ob wir es bei psychischen Erkrankungen mit natürlichen Entitäten (natural kinds) - Emil Kraepelin sprach von "natürlichen Krankheitseinheiten» - zu tun haben, oder ob es sich letztlich immer, auch bei den psychischen Krankheiten im Sinne des Autors, um begriffliche Konstrukte handelt, die nicht auf ein ontologisches Fundament verweisen müssen.

Ich möchte die zuletzt genannten Fragen an das Buch nicht missverstanden wissen: Es geht mir keinesfalls um eine grundsätzliche Kritik, sondern um das, wozu der Autor uns auffordert, nämlich mit aufklärerischer

\section{Die Argumentation läuft auf eine drastische} Reduktion der Zahl psychischer Erkrankungen hinaus.

Grundhaltung und patientennaher Herangehensweise alle nosologischen Modelle in der Psychiatrie kritisch zu hinterfragen, also auch das von ihm selbst postulierte. Unser Fach ist es seinen Patientinnen und $\mathrm{Pa}$ tienten, aber auch sich selbst schuldig, komplexe theoretische Fragen immer wieder neu aufzuwerfen und mit dem je aktuellen Stand empirischen Wissens zu verschränken. Wer sich diesen Fragen ernsthaft stellen will, die sich jenseits des Tellerrandes einer eingeschliffenen Praxis auftun, wird das Buch mit grossem Gewinn lesen. Er oder sie wird es allerdings nach der Lektüre auch nachdenklich zur Seite legen. Genau das bringt die Psychiatrie weiter. 